\title{
FRECUENCIA DE PARÁSITOS GASTROINTESTINALES EN ÉQUI- DOS DESTINADOS PARA CONSUMO HUMANO EN UNA PLANTA DE BENEFICIO EN ANTIOQUIA, COLOMBIA.
}

\author{
Ana L. Castaño González¹; León D. Ramírez Arias²; María José Casas Soto³. \\ 1 Docente Facultad de Medicina Veterinaria de la Corporación Universitaria Remington, Calle 51 No 51-27 Edifi- \\ cio Remington. E-mail: ana.castano@uniremington.edu.co. \\ ${ }^{2}$ Médico Veterinario de la Corporación Universitaria Remington, Calle 51 No 51-27 Edificio Remington. E-mail: \\ león. ramirez3516@miremington.edu.co \\ ${ }^{3}$ Médica Veterinaria de la Corporación Universitaria Remington, Calle 51 No 51-27 Edificio Remington. E-mail: \\ majovet4444@gmail.com
}

\begin{abstract}
RESUMEN
Existen pocos reportes de la frecuencia de parásitos gastrointestinales en equinos faenados para consumo humano en Colombia, por esta razón fueron muestreados 1050 equinos que ingresaron a una planta de beneficio en Antioquia, con el fin de determinar la frecuencia de parásitos gastrointestinales que más afecta a los équidos. La muestra fue tomada directamente del recto con todas las medidas higiénico sanitarias. A cada muestra de materia fecal se le realizó examen coprológico a través de la técnica de flotación con solución sobresaturada de $\mathrm{NaCl}$. Del $100 \%$ de los animales evaluados no fue posible saber si contaban o no con plan sanitario ya que la gran mayoría de équidos ingresaban a la planta por mercaderes los cuales no registraban ninguno de estos datos, por lo que se registraron sin plan sanitario. Los parásitos encontrados con mayor frecuencia fueron: Strongylos $s p(72,1 \%)$, seguido por Entamoeba histolytica (1,0\%), Endolimax nana (0,9\%), Parascaris sp (0,8\%), Oxyuris sp (0,8\%), Entamoeba coli $(0,3 \%)$, y Blastocystis hominus $(0,2 \%)$. Siendo la mayor cantidad de caballos provenientes de Córdoba (71\%), seguido por Antioquia (23\%), Bolívar (3,0\%) y por último Sucre (2,7\%). De la cantidad de animales muestreados 995 eran caballos y 55 asnales, además se evidenció que las hembras presentaban un mayor porcentaje $53.2 \%$ respecto a los machos $46.8 \%$.
\end{abstract}

Palabras clave: Equinos ${ }^{1}$, Asnos $^{2}$, Parásitos ${ }^{3}$, Protozoarios ${ }^{4}$, Strongyls $s p^{5}$, Planta de beneficio ${ }^{6}$.

Recibido: 17 de Abril de 2020. Aceptado: 9 de Diciembre de 2020

Received: April 17, 2020. Accepted: December 9, 2020

\section{FREQUENCY OF GASTROINTESTINAL PARASITES IN EQUIPMENT INTENDED FOR HUMAN CONSUMP- TION IN A SLAUGHTERHOUSE PLANT IN ANTIOQUIA, COLOMBIA.}

\begin{abstract}
There are few reports of the prevalence of gastrointestinal parasites in equines slaughtered for human consumption in Colombia, for this reason 1050 equines that entered a slaughter plant in eastern Antioquia were sampled, in order to determine the incidence of gastrointestinal parasites that most affects For equines, each stool sample underwent a stool examination accompanied by the floatation technique with supersaturated $\mathrm{NaCl}$ solution, as it is fast, provides good results and is also easy to prepare, in addition to being the most common, simple and cheap $100 \%$ of the animals evaluated did not present a health plan, the parasites most frequently found were: Strongylos $s p(72.1 \%)$, followed by Entamoeba histolytica (1.0\%), Endolimax nana (0.9\%), Parascaris sp (0.8\%), Oxyuris $s p$ (0.8\%), Entamoeba coli (0.3\%), and Blastocystis hominus (0.2\%). Being the largest number of horses from Córdoba (71\%), followed by Antioquia (23\%), Bolívar (3.0\%) and finally Sucre (2.7\%). Of the number of animals sampled, 995 were horses and 55 donkeys, and it was also shown that females presented a higher percentage of $53.2 \%$ compared to males, $46.8 \%$.
\end{abstract}

Keywords: Equines ${ }^{1}$, Donkeys $^{2}$, Parasites ${ }^{3}$, Protozoa ${ }^{4}$, Strongylus $s p^{5}$, Slaughterhouseadec ${ }^{6}$

Cómo citar este artículo: A. Castaño L. Ramírez, M. Casas. "Frecuencia de parásitos gastrointestinales en équidos destinados para consumo humano en una planta de beneficio en Antioquia, Colombia". Revista Politécnica, vol.16, no.32 pp.31-40, 2020. DOI:10.33571/rpolitec.v16n32a3 


\section{INTRODUCCIÓN}

En la actualidad, los equinos cuentan con cualidades como velocidad, fuerza, inteligencia, nobleza, entre otras características, lo que permite que sean utilizados en actividades tales como transporte de personas y mercancías, ayuda en labores agrícolas y ganaderas, actividades deportivas y turísticas, y ocasionalmente como mascotas.

El consumo de carne de caballo en la actualidad no es popular en la mayoría de los países, pero debido a su disponibilidad y valor nutricional reconocido, el consumo está aumentando lentamente en varios países de Europa occidental basándose en las afirmaciones de que podría ser una alternativa a la carne roja proveniente de otras especies como lo son actualmente los bovinos. (Belanzauran, y otros, 2015).

Según estadísticas de la FAO en América del sur los productores de carne de caballo son, Argentina (25.970 ton), Brasil (21.800 ton), seguidos por Chile (8.324 ton), Uruguay (8.170 ton), Colombia (6.750 ton) y Paraguay (3.960 ton).

La transmisión de enfermedades a través del consumo de alimentos es un fenómeno ya conocido; sin embargo, recientemente la frecuencia de casos clínicos relacionados con agentes infecciosos viene en aumento debido a cambios en las etiologías predominantes y en la dinámica epidemiológica (Worku \& Afera, 2012).

El decreto 2278 de agosto 2 de 1982 del Ministerio de Salud, declara a los équidos como animales de consumo humano para efectos de exportación. La resolución 00222 de enero 17 de 1990 del Ministerio de Salud, declara aptos los equinos como animales de abasto público en el Territorio Nacional, considerando esto actualmente hay un volumen considerable de equinos que son destinados a la producción de carne, la información relacionada con datos de producción, comercialización y consumo es muy limitada.

En Colombia la cadena de producción de carne equina no se encuentra estandarizada y su consumo se ha visto limitado debido a factores como: 1. falta de sistemas de producción especializados, lo que se traduce en el sacrificio de animales que generalmente han terminado su vida productiva o que sufren algún tipo de lesión; 2. prácticas ilegales de sacrificio y ausencia de trazabilidad de los animales destinados a sacrificio, lo que conlleva a incrementar la desconfianza del consumidor sobre la calidad de este tipo de carne; y 3 . factores sociales y culturales, pues el equino es considerado un animal doméstico destinado a otras actividades. (Nivia \& Vanegas-Azurero, 2014) Esta situación requiere más información sobre el estado sanitario de los équidos y las medidas adecuadas para garantizar que estén en una condición física adecuada para su sacrificio y posterior comercialización y consumo.

Las patologías que afectan a esta especie son variables y se clasifican según la etiología que las desencadena. Los parásitos intestinales pueden afectar negativamente algunos índices de la producción mundial equina (trabajo, ganancia de peso, recreación y reproducción) hasta en un $24 \%$, así lo sugiere un estudio epidemiológico que se desarrolló en la Universidad Nacional de Río Cuarto, en Argentina. Es de suma importancia mantener a los animales en óptimas condiciones de salud y nutrición debido a que hay más de 150 tipos de parásitos que se pueden manifestar con diferentes signos, como: pérdida de peso, letargo, disminución de la resistencia, fiebre, anemia, retraso en el crecimiento, pelaje sin brillo, diarrea, cólico y muerte (Bedoya Ríos, Arcila Quiceno, Díaz Arias, \& Reyes Plata, 2011).

Los principales parásitos internos que afectan a los caballos se pueden agrupar en dos grupos: céstodos (Anoplocephala spp) y nemátodos (Paráscaris equorum, estróngilos grandes y pequeños, Oxyuros equi, Strongyloides westeri y Dictyocaulus arnfieldi) (Dwight D, 2004). La prevalencia de infecciones por nemátodos gastrointestinales en equinos se ha reportado en rangos entre el 22,9 y el $100 \%$ en diferentes regiones del mundo. (Chaparro Gutiérrez, y otros, 2018). En otro estudio realizado en la sabana inundable del municipio de Arauca, Colombia se encontró que el mayor porcentaje de infestación fue para Strongylus $s p(98,53 \%)$, seguido de Strongyloides $s p(0,48 \%)$, Habronema $s p(0,40 \%)$, Oxyuris $s p$ $(0,32 \%)$ y Paranoplocephala sp $(0,24 \%)$ (Moreno, Salamanca Quintero, \& Arenas, 2015).

medida que la solidez del rotor se hace más grande, y el rendimiento del rotor es casi similar cuando se determina que TSR y la solidez del rotor tienen el mismo valor. 
Una de las maneras de diagnosticar las parasitosis gastrointestinales es mediante la aplicación de técnicas coproparasitológicas de enriquecimiento (sedimentación y flotación), que permiten determinar su presencia e identificarlos correctamente (Navone, y otros, 2005). El desconocimiento o la falta de uso de estas técnicas llevan al uso indiscriminado y extensivo de antihelmínticos, generando gran preocupación por las múltiples resistencias que han surgido ante estos medicamentos, haciendo reconsiderar estas prácticas al momento de instaurar un tratamiento antiparasitario (Corning, 2009).

\section{MATERIALES Y METODO}

\section{Tipo de estudio}

Se realizó un estudio Descriptivo.

\section{Aspectos éticos}

En el desarrollo de esta investigación, se manejaron políticas de confidencialidad con respecto al uso de la información obtenida.

La obtención de la muestra no representó daño alguno para el animal, ya que fue recolectada en un procedimiento de mínima invasión y aplicando las normas de bioseguridad, velando por el bienestar de los équidos sin considerar su destino.

\section{Población y tamaño de la muestra}

Este estudio se efectuó en una planta de beneficio equino al oriente de Antioquia, a esta planta ingresan equinos de diferentes regiones del país; Córdoba, Antioquia, Bolívar y Sucre. El tamaño de muestra fue de 1050 équidos en los que se comprende caballos y asnos que ingresaron a la planta en un periodo de mayo y junio del 2018, la muestra fue escogida al azar.

\section{Toma de muestra y pruebas de laboratorio}

Los médicos veterinarios pertenecientes a la investigación realizaron la toma de muestra de materia fecal directamente del recto de los animales estudiados, tomando en consideración las medidas de bioseguridad necesarias para evitar la contaminación del profesional, manteniendo la integridad del material recolectado y evitando el entrecruzamiento de material biológico entre los animales. El volumen fue 10 gramos de materia fecal aproximadamente, la muestra fue rotulada con un número (consecutivo), almacenada en nevera de icopor y sometida a refrigeración (entre 4 y $10^{\circ} \mathrm{C}$ ), la temperatura fue monitoreada utilizando un termómetro desde la toma de la muestra hasta su análisis en el laboratorio.

Las muestras obtenidas en la planta de beneficio se analizaron dentro de las siguientes 10 horas, con el fin de evitar que los parásitos murieran una vez estuvieran fuera de su hospedador (Équido). Al llegar al laboratorio de La Vida de la Corporación Universitaria Remington, las muestras de laboratorio fueron procesadas por un(a) Bacteriólogo(a), a través de metodología de montaje directo de las heces (coprológico) donde se incluye el examen macroscópico (consistencia, color, presencia de elementos no fecales, moco o tejido intestinal) y un análisis microscópico donde previamente se realizó la técnica de flotación con solución sobresaturada de $\mathrm{NaCl}$, por ser rápida, brindar buenos resultados y también por la facilidad de preparación de la solución, además de ser la más común, sencilla y económica.

\section{RESULTADOS}

Fueron muestreados 1050 équidos (caballares y asnales) en una planta de beneficio destinados para consumo humano en los meses de mayo y junio del 2018, con el fin de determinar la frecuencia de parásitos gastrointestinales que más los afectan.

La frecuencia de parásitos gastrointestinales corresponde al 75,8\% que equivale a 796 animales, el $24,2 \%$ de los animales resultaron negativos a la presencia de parásitos gastrointestinales (Tabla 1). 
Tabla 1. Frecuencia de parásitos gastrointestinales en équidos faenados en el oriente de Antioquia, Colombia.

\begin{tabular}{lcc}
\hline & $\begin{array}{c}\text { Frecuencia } \\
\text { absoluta }\end{array}$ & $\begin{array}{c}\text { Frecuencia } \\
\text { relativa }\end{array}$ \\
\hline $\begin{array}{l}\text { Muestras } \\
\text { positivas }\end{array}$ & 796 & $75.8 \%$ \\
$\begin{array}{l}\text { Muestras } \\
\text { negativas }\end{array}$ & 254 & $24.2 \%$ \\
Total & 1050 & $100 \%$ \\
\hline
\end{tabular}

Se obtuvo que el $100 \%$ de los animales evaluados no reportaron plan sanitario, el $(71 \%)$ de estos provenían del departamento de Córdoba, seguido por Antioquia (23.3\%), Bolívar (3\%), y por último Sucre (2,7\%) (Tabla 2).

Tabla 2. Procedencia de animales faenados en el oriente de Antioquia, Colombia.

\begin{tabular}{lcc}
\hline Departamento & $\begin{array}{c}\text { Frecuencia } \\
\text { absoluta }\end{array}$ & $\begin{array}{c}\text { Frecuencia } \\
\text { relativa }\end{array}$ \\
\hline Córdoba & 745 & $71 \%$ \\
Antioquia & 245 & $23.3 \%$ \\
Bolívar & 32 & $3 \%$ \\
Sucre & 28 & $2.7 \%$ \\
Total & 1050 & $100 \%$ \\
\hline
\end{tabular}

Se obtuvo que el departamento que más équidos parasitados ingresó a la planta fue Bolívar ya que el $91 \%$ se encontraban parasitados, seguido de Sucre con el $82 \%$, Córdoba con el $76 \%$ y por último Antioquia con el $72 \%$ (Tabla 3).

Tabla 3. Frecuencia de équidos parasitados por regiones.

\begin{tabular}{lcccccc} 
& F.P & $\%$ P & F.N.P & $\%$ N.P & F. T & $\%$ AC \\
\hline Bolívar & 29 & $91 \%$ & 3 & $9 \%$ & 32 & $100 \%$ \\
Córdoba & 566 & $76 \%$ & 179 & $24 \%$ & 745 & $100 \%$ \\
Antioquia & 177 & $72 \%$ & 68 & $28 \%$ & 245 & $100 \%$ \\
Sucre & 23 & $82 \%$ & 5 & $18 \%$ & 28 & $100 \%$ \\
Total & 795 & & 255 & & 1050 & $100 \%$ \\
\hline
\end{tabular}

F.P: Frecuencia parasitados

$\%$ P: Porcentaje parasitados

F.N.P: Frecuencia no parasitados

$\%$ N.P: Porcentaje no parasitados

F.T: Frecuencia total

$\%$ AC: Porcentaje acumulado

En la distribución por especie puede apreciarse que 995 de estos eran caballos, y 55 asnales (Tabla 4), además se evidenció que las hembras eran quienes ingresaban con mayor frecuencia a la planta $53,2 \%$ con relación a los machos $46,8 \%$. (Tabla 5). 
Tabla 4. Distribución por especie de animales faenados en el oriente de Antioquia, Colombia.

\begin{tabular}{lcc}
\hline Especie & $\begin{array}{c}\text { Frecuencia } \\
\text { absoluta }\end{array}$ & $\begin{array}{c}\text { Frecuencia } \\
\text { relativa }\end{array}$ \\
\hline Caballar & 995 & $94.8 \%$ \\
Asnal & 55 & $5.2 \%$ \\
Total & 1050 & $100 \%$ \\
\hline
\end{tabular}

Tabla 5. Distribución del sexo en animales faenados en el oriente de Antioquia, Colombia.

\begin{tabular}{lcc}
\hline Sexo & $\begin{array}{c}\text { Frecuencia } \\
\text { absoluta }\end{array}$ & $\begin{array}{c}\text { Frecuencia } \\
\text { relativa }\end{array}$ \\
\hline Macho & 491 & $46.8 \%$ \\
Hembra & 559 & $53.2 \%$ \\
Total & 1050 & $100 \%$ \\
\hline
\end{tabular}

De las 1050 muestras evaluadas se detectaron huevos de Strongyluos sp (72,1\%), seguido por quistes de Entamoeba histolytica (1,0\%), quistes de Endolimax nana $(0,9 \%)$, huevos de Parascaris $s p$ $(0,8 \%)$, huevos de Oxyuros sp $(0,8 \%)$, quistes de Entamoeba coli $(0,3 \%)$ y quistes de Blastocystis hominus $(0,2 \%)$. (Tabla 6$)$.

Tabla 6. Distribución de parásitos encontrados en animales faenados en el oriente de Antioquia, Colombia.

\begin{tabular}{lcc}
\hline Agente & $\begin{array}{c}\text { Frecuencia } \\
\text { absoluta }\end{array}$ & $\begin{array}{c}\text { Frecuencia } \\
\text { relativa }\end{array}$ \\
\hline Strongilos sp. & 756 & $72 \%$ \\
Entamoeba his- & 10 & $0.95 \%$ \\
tolytica/dispar & & \\
Endolimax nana & 9 & $0.85 \%$ \\
Parascaris sp. & 8 & $0.76 \%$ \\
Oxyuros sp. & 8 & $0.76 \%$ \\
Entamoeba coli & 3 & $0.28 \%$ \\
Blastocistys ho- & 2 & $0.2 \%$ \\
minus & & \\
Total & 796 & $75.8 \%$ \\
\hline
\end{tabular}

El número de animales parasitados con Strongylus sp. con respecto a su procedencia, se obtuvo que 545 procedían de Córdoba, 163 de Antioquia, 29 de Bolívar y 19 de Sucre. (Grafica 1); El número de animales parasitados con Strongylos $s p$ con respecto al sexo, se obtuvo que 353 ejemplares eran machos y 403 hembras. (Grafica 2). 
Grafica 1. Número de animales parasitados con Strongylos sp. respecto a su procedencia.

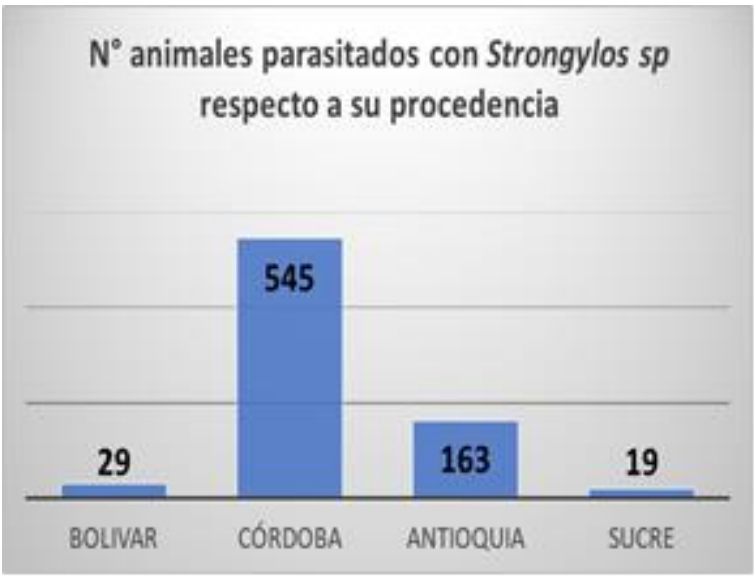

En el gráfico 2 se puede ver que no hay diferencia significativa entre las proporciones de machos y hembras con presencia de Strongylos.

Grafica 2. Número de animales parasitados con Strongylos sp. con respecto al sexo.

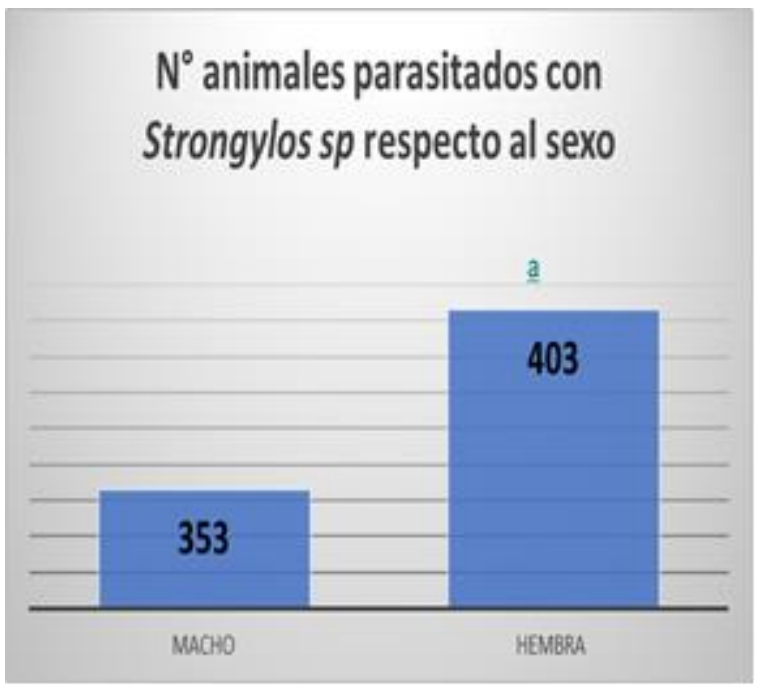

Para el cálculo de las diferencias entre las proporciones se empleó el método hibrido de Newcombe.

En la tabla 7. se puede ver como existió diferencia significativa (95\% de confiabilidad), para las diferencias entre todas las prevalencias excepto entre Sucre y Antioquia, y entre Sucre y Córdoba.

Tabla 7. Intervalos de confianza para la diferencia entre las prevalencias de Strongylos. sp entre los distintos orígenes de los équidos.

\begin{tabular}{|c|c|c|c|c|}
\hline PRO & Bolívar & Córdoba & Antioquia & Sucre \\
\hline Bolívar & & 0.0280 .25 & 0.0960 .344 & 0.0220 .423 \\
\hline Córdoba & & & 0.0040 .143 & -0.1020 .23 \\
\hline Antioquia & & & & -0.1840 .166 \\
\hline
\end{tabular}

Fuente: Elaboración propia, 2020 
En la siguiente tabla se puede ver que solo se detectó diferencia significativa entre Sucre-Antioquia y entre Sucre y Córdoba.

Tabla 8. Intervalos de confianza para la diferencia entre las prevalencias de Parascaris entre los distintos orígenes de los équidos.

\begin{tabular}{|c|c|c|c|c|}
\hline PRO & Bolívar & Córdoba & Antioquia & Sucre \\
\hline Bolívar & & 0.0280 .25 & -0.0240 .103 & \\
\hline Córdoba & & & -00190011 & -0.2720 .021 \\
\hline rotuda & & & -0.0190 .011 & $-0.267-0.031$ \\
\hline Antioquia & & & & $-0.268-0.03$ \\
\hline Sucre & & & & \\
\hline
\end{tabular}

Fuente: Elaboración propia, 2020

\section{DISCUSIÓN}

Este estudio abarcó el mayor número de équidos muestreados para coprológico y flotación en Colombia. Reveló que el $75,8 \%$ de los animales estaban parasitados y un $24,2 \%$ se encontraban negativos a parásitos gastrointestinales. Los Strongylus sp. fueron parásitos más frecuentes que afectan a los équidos faenados en el oriente de Antioquia. Es importante considerar que ninguno de los animales que llegan para el faenado, en el caso de esta planta hace reporte sobre el plan sanitario, por lo tanto, no se sabe si está vigente o no, sin embargo, por lo encontrado en esta investigación la mayoría de los especímenes biológicos se encontraban parasitados, no se esclareció para este caso la carga parasitaria.

La frecuencia de parásitos gastrointestinales encontrada en el estudio significa que en general es pobre el estado sanitario de dichos ejemplares, ya que en estos se observó estados de baja condición corporal, pelo hirsuto, entre otros indicadores de posibles parasitosis, que afectan su rendimiento metabólico y deterioran su salud. Además, es de resaltar que el mayor número de animales parasitados con Stron ylus sp provenían del departamento de Bolívar (91\%), esto genera la necesidad de evaluar el manejo sanitario de los équidos en esta región.

En el presente estudio se evidenció que los Strongylus sp. fue el principal parásito gastrointestinal hallado, representando el $72,1 \%$ de los casos es decir 756/1050 de los équidos muestreados. En una investigación reciente realizada en equinos del departamento de Antioquia, Colombia, se reveló que los Strongylus representan el grupo de parásitos más frecuente que infecta a los equinos de Antioquia, con una prevalencia del $54 \%$, es decir en 360 de los 664 animales (Chaparro Gutiérrez, y otros, 2018).

La Estrongiloidosis es una parasitosis cosmopolita presente en países de clima tropical o templado, especialmente de Asia, África y Latinoamérica, afecta a diversas especies mamíferas incluido el hombre; los Strongylus $s p$ que afectan al équido pueden llegar al humano causando la patología conocida como larva migrans cutánea (larva migratoria cutánea) la cual ocurre cuando las larvas parasitarias migran a través de la piel de un hospedador, esta infección se contrae cuando se expone a un medio ambiente (suelo o aguas) contaminadas con materia fecal de los animales portadores. Las larvas causan una dermatitis migratoria pruriginosa a medida que viajan por la piel.

Las tres principales especies de grandes Strongylos del equino son: S. vulgaris, S. edentatus y S. equinus; El S. vulgaris es el más patógeno y frecuente. Algunos estudios realizados señalan que el $S$. vulgaris presenta una prevalencia del 80,5\% (Kornas, Skalska, Nowosad, Gawor, \& Kharchenko, 2009).

La evidencia de protozoarios específicos de humanos que se obtuvo en el estudio podría indicar el mal uso o descarte de aguas residuales por parte de la población humana lo que conlleva a que los équidos también se encuentren parasitados por estos agentes. No se ha encontrado investigaciones recientes que indiquen la presencia de protozoarios en équidos para el caso de Colombia. Es relevante generar nuevos estudios que se propongan evaluar dichos agentes y su importancia en la salud pública.

El protozoario que se aisló con mayor frecuencia fue la Entamoeba histolytica/dispar representando el 1,0\% de los 1050 equinos muestreados, es decir $10 / 1050$ equinos la presentaban, esta es una de las principales 
causas de diarrea en humanos menores de cinco años debido a infección por protozoarios (Gómez, Cortés, Cuervo, \& López, 2007), al igual que la Blastocystis hominus (0,2\%) aunque este protozoo fue hallado en menor cantidad es responsable de causar procesos diarreicos en los seres humanos (Salinas \& Gonzales, 2007)

La Endolimax nana y Entamoeba coli representaron el 0,9\% y el 0,3\% respectivamente, es decir $9 / 1050$ y 3/1050 equinos contenían estos protozoarios. Estos, según la literatura se consideran no patógenos, sin embargo, la Endolimax nana se reporta habitante del tracto gastrointestinal humano, es decir, se reitera la problemática del manejo de aguas residuales procedentes de las poblaciones humanas y su subsecuente llegada a los animales. Es relevante indagar en investigaciones futuras acerca del destino final y proceso de trazabilidad de las vísceras que son extraídas de los caballos y usadas para embutidos de consumo humano.

Es de importancia resaltar la ausencia de céstodos en esta investigación que puede deberse a que el hospedador intermediario de las Cestodosis digestivas equinas es el ácaro (Galumma spp) y que dicho hospedador depende de la estacionalidad (temperatura y humedad), por lo que se especula que en el período muestreado la presencia del vector era baja y en ese orden de ideas la magnitud de la infección por Céstodos no fue relevante en los animales estudiados. (Cordero del Campillo \& Rojo Vázquez, 2000). Otra posibilidad que podría considerarse es la baja prevalencia de Céstodos en Antioquia como lo sugiere el estudio de Chaparro y colaboradores que reportan un $0,5 \%$ de animales positivos a Anoplocephala sp. (Chaparro Gutiérrez, y otros, 2018) esto también puede deberse al uso indiscriminado de antiparasitarios a los cuales presuntamente pueden haber generado resistencia los nematodos y para el caso de los céstodos esta no sea su condición, se requieren más estudios para esclarecer esta condición.

Los programas de Salud Pública Veterinaria en los países en vías de desarrollo deben evolucionar en complejidad técnica y alcance a medida que se desarrollan en el tiempo. Lo ideal sería el control de enfermedades zoonóticas y la implementación de programas de inocuidad alimentaria que eduquen al individuo en métodos y prácticas que pueden ser llevados a cabo con bajos costos y con el mínimo de equipamiento y materiales, siendo el principio básico el dirigir los programas de salud pública directamente al individuo, grupos pequeños o comunidades (Sandoval Martínez, 2011).

\section{CONCLUSIONES}

La frecuencia de parásitos gastrointestinales en équidos faenados es de 75,8\% siendo este valor de importancia clínica y sanitaria. Se debe esclarecer en futuras investigaciones la carga parasitaria en contraste con la presencia de parásitos. También se debe esclarecer en futuras investigaciones el impacto que tiene esta parasitosis sobre los márgenes productivos del équido, estableciendo si para el proceso de producción de carne la rentabilidad es poca rentabilidad o no después del de faenado.

Los équidos son considerados una fuente de carne de buena calidad proteica y bajo costo, sin embargo, el manejo de los animales previo al faenado está en detrimento de las ganancias y el bienestar animal. Estos animales no reportan planes sanitarios y de manejo acordes a su destino final, incurriendo en una falla del proceso de trazabilidad, por tanto, el consumo de esta carne esta mitificado como consumo de carne de mala calidad. Este estudio evidencia la importancia de estrategias de buenas prácticas para quienes pretenden la comercialización de animales que tendrán como destino final el consumo por parte de la población colombiana.

Cabe descartar la presencia de agentes protozoarios específicos de humanos, aunque es en bajos porcentajes Entamoeba histolytica/dispar (1,0\%), Endolimax nana (0,9\%), Entamoeba coli $(0,3 \%)$, Blastocystis hominus $(0,2 \%)$, nos permite establecer dos puntos importantes, el primero se deriva del mal manejo de las aguas residuales y la factibilidad de que estén llegando al agua de bebida o pastos destinados para los animales y el segundo punto es la interacción de estos protozoarios con hospedadores que no son naturales, es decir, que estos agentes están traspasando las barreras biológicas de especies ajenas a su ciclo vital y generando posible diseminación. Aunque no podemos asegurar que los équidos estén siendo afectados por estos protozoarios, pues no hay signos clínicos que lo confirmen o descarten, el pasaje de estos microorganismos por el digestivo del équido sugiere adaptación del parásito y la posibilidad futura de diseminación de patógenos a la población vinculada con estos animales. 
Finalmente es relevante proponer estudios que permitan la identificación exacta (género y especie) ya sea por taxonomía o por biología molecular, de los parásitos presentes en los équidos destinados para consumo humano, ya que con esta información se establecerían estrategias de manejo, prevención y tratamiento orientados al agente patógeno especifico, mejorando la calidad de vida del animal y sus parámetros productivos.

Las conclusiones son obligatorias y deben ser claras. Su contenido no debería duplicar substancialmente el resumen. Deben expresar el balance final de la investigación o la aplicación del conocimiento o temática tratada. Se discute sobre las implicaciones del estudio y la relevancia que tiene para el área del conocimiento. Se sugiere no concluir más cosas de las que los resultados permitan. En esta sección se suelen mencionar también los trabajos futuros que se pueden realizar en el tema.

\section{AGRADECIMIENTOS}

Agradecemos a la Corporación Universitaria Remington por el apoyo financiero y académico para desarrollar esta investigación, a los operarios y área administrativa de la planta de beneficio por abrirnos sus puertas y permitirnos llevar este proyecto hasta su terminación.

Gracias a todas las personas que de forma directa e indirecta aportaron al desarrollo de este proyecto.

\section{REFERENCIAS BIBLIOGRÁFICAS}

[1] Belanzauran, X., Bessa, R., Lavín, P., Matecón, A., Kramer, J., \& Aldai, N. (2015). Horse-meat for human consumption - Current research and future opportunities. Elsevier Ltd, 74 - 81.

[2] Worku, S., \& Afera, B. (2012). Prevalencia de nematóodos en equinos en y alrededor del sur de Wollo Kombolcha, Ethiopia. REDVET, 14.

[3] Nivia, A., \& Vanegas-Azurero, A. M. (2014). Caracterización del sistema de comercialización de animales y carne de la especie equina en torno a una planta de beneficio. Ciencia Animal, 95 - 110.

[4] Bedoya Ríos, M. Á., Arcila Quiceno, V. H., Díaz Arias, D. A., \& Reyes Plata, E. A. (2011). Prevalencia de parásitos gastrointestinales en équidos del municipio de Oiba (Santander). REVISTA SPEI DOMUS, 7.

[5] Dwight D, B. (2004). Parasitología para veterinarios. Madrid: Elsevier Edición 8.

[6] Chaparro Gutiérrez, J. J., Ramírez Vásquez, N. F., Piedrahita, D., Strauch, A., Sánchez, A., Tobón, J., . . Villar Argaiz, D. (2018). Prevalencia de parásitos gastrointestinales en equinos y factores de riesgo asociados en varias zonas de Antioquia, Colombia. CES Medicina Veterinaria y Zootecnia, 10.

[7] Moreno, Y., Salamanca, A., Quintero, A. D., \& Arenas, M. J. (2015). agentes parasitarios presentes en el tracto gastrointestinal de caballos criollos de la sabana inundable del municipio de Arauca, Colombia. Actas Iberoamericanas de Conservación Animal, 150 - 155.

[8] Navone, G. T., Gamboa, M. I., Kozubky, L. E., Costas, M. E., Cardozo, M. S., Sisliauskas, M. N., \& González, M. (2005). Estudio comparativo de recuperación de formas parasitarias por tres diferentes métodos de enriquecimiento coproparasitológico. Parasitol Latinoam, 4.

[9] Corning, S. (2009). Equine cyathostomins: a review of biology, clinical significance and therapy. BioMed Central, $1-6$.

[10] Sandoval Martínez, L. E. (2011). Consumo de carne de equino en Bogotá y su impacto en la salud pública. Universidad de ciencias aplicadas y ambientales. 
[11] Chaparro Gutiérrez, J. J., Ramírez Vásquez, N. F., Piedrahita, D., Strauch, A., Sánchez, A., Tobón, J., . .. Villar Argaiz, D. (2018). Prevalencia de parásitos gastrointestinales en equinos y factores de riesgo asociados en varias zonas de Antioquia, Colombia. CES Medicina Veterinaria y Zootecnia, 10.

[12] Kornas, S., Skalska, M., Nowosad, B., Gawor, J., \& Kharchenko, V. (2009). Occurrence of strongyles (Strongylidae) in horses from small farms on the basis of necropsy. Infectiologie Animale et Santé Publique.

[13] Gómez, J. C., Cortés, J. A., Cuervo, S. I., \& López, M. C. (2007). Amebiasis intestinal. Asociación colombiana de infectologíia, 10.

[14] Salinas, J. L., \& Gonzales, H. V. (2007). Infección por Blastocystis. Revista de Gastroenterología del Perú, 11.

[15] Cordero del Campillo, M., \& Rojo Vázquez, F. A. (2000). Paraásitologíia veterinaria España: McGraw-Hill Interamericana de España.

[16] Chaparro Gutiérrez, J. J., Ramírez Vásquez, N. F., Piedrahita, D., Strauch, A., Sánchez, A., Tobón, J., . . . Villar Argaiz, D. (2018). Prevalencia de parásitos gastrointestinales en equinos y factores de riesgo asociados en varias zonas de Antioquia, Colombia. CES Medicina Veterinaria y Zootecnia, 10. 